\title{
Role of Gastrin Supersensitivity in the Pathogenesis of
}

\section{Lower Esophageal Sphincter Hypertension in Achalasia}

\author{
Sidney Cohen, William Lipshutz, and William Hughes \\ From the Gastrointestinal Section, Department of Medicine of the University \\ of Pennsylvania at the Hospital of the University of Pennsylvania and Veterans \\ Administration Hospital, Philadelphia, Pennsylvania 19104
}

A B S T R A C T Intraluminal manometric studies were carried out in 19 patients with untreated achalasia and in 20 normals. Lower esophageal sphincter (LES) pressure was $50.5 \pm 4.6 \mathrm{~mm} \mathrm{Hg}$ in patients with achalasia as compared with $19.4 \pm 1.3 \mathrm{~mm} \mathrm{Hg}$ in the normal group. In both groups, the LES pressure was lowered when exogenous $0.1 \mathrm{~N} \mathrm{HCl}$ was placed into the stomach. Although the nadir of pressure attained with acid suppression was the same, the per cent inhibition was significantly greater in patients with achalasia. Serum gastrin levels were the same in the two groups studied. The patients with achalasia, pre- and postpneumatic dilatation, showed a supersensitivity to exogenous intravenous gastrin I, as compared with normals. These data suggest that high, acid-suppressible levels of LES pressure, in patients with achalasia, are due to supersensitivity to endogenous gastrin.

\section{INTRODUCTION}

In patients with achalasia, dysfunction of the lower esophageal sphincter $(\mathrm{LES})^{1}$ plays a major role in the pathogenesis of impaired esophageal emptying. Although this point is well recognized, a clear understanding of the pathophysiology of this dysfunction is not available. The classic concept of a normotensive LES, which fails to relax upon deglutition $(1,2)$ has been questioned (3). It has been suggested that LES dysfunction consisted of a marked elevation in LES pressure and a limited, but distinct, ability to relax upon deglutition. By this latter concept, LES hypertension would play a major role in the impairment of esophageal emptying. It is the purpose of this study to utilize a method of proven

\footnotetext{
A portion of this work was presented before the plenary session of the American Federation for Clinical Research, Midwestern Section, Chicago, Ill. 5 November 1970.

Received for publication 4 November 1970.

${ }^{1}$ Abbreviation used in this paper: LES, lower esophageal sphincter.
}

validity (4-6) to quantitate LES pressure in patients wiih achalasia, and to define the role of gastrin in the $p^{\sim}$ thogenesis of LES hypertension.

\section{METHODS}

Three water-filled polyvinyl catheters, $1.4 \mathrm{~mm}$ internal diameter, were used to transmit intraluminal pressures to external transducers (Statham P23BB). Output from these transducers was recorded on a multichannel Beckman curvilinear ink-writing recorder. The recording catheters were arranged to measure intraluminal pressure at three points, $5 \mathrm{~cm}$ apart, through side openings $1.2 \mathrm{~mm}$ in diameter. Gastric infusion was done through a fourth catheter which had multiple perforations over the $8 \mathrm{~cm}$ distal segment. The four polyvinyl catheters were joined into a single unit with an outside diameter of $5 \mathrm{~mm}$. The midpoint of the multiperforated area was $16 \mathrm{~cm}$ distal to the middle recording orifice.

Studies were performed on two groups of patients with informed consent obtained from each patient. The first group consisted of 19 patients, aged 10-71 yr (mean, 46), with well-defined, untreated achalasia. The diagnostic criteria satisfied in each patient before inclusion in this group were the following: (a) radiographic and cineradiographic diagnosis of achalasia by a radiologist, independent of the investigators; $(b)$ manometric recording showing total absence of distally progressive peristalsis; (c) endoscopic evaluation with free passage of the esophagoscope into the stomach, ruling out organic obstruction; and $(d)$ a positive response to acetyl- $\beta$-methacholine (Mecholyl chloride), ${ }^{2} 2-10$ mg subcutaneously, manifested as an increase in esophageal pressure of over $20 \mathrm{~mm} \mathrm{Hg}$, which was recorded by opentipped catheters. A second group of 20 patients, aged 22-76 yr (mean, 44), with normal upper gastrointestinal radiographic evaluations, were used as controls. No patient had undergone upper gastrointestinal tract surgery. All patients were studied while resting quietly in the supine position after an $8 \mathrm{hr}$ fasting period. Belt pneumographs around the chest and over the larynx were used to monitor respiration and swallowing, respectively. The recording assembly was positioned with all orifices in the stomach. After a $20 \mathrm{~min}$ rest period, the assembly was moved at $1 \mathrm{~cm}$ intervals through the full length of the esophagus. After this diag-

\footnotetext{
${ }^{2}$ Merck \& Co., Inc., Rahway, N. J.
} 


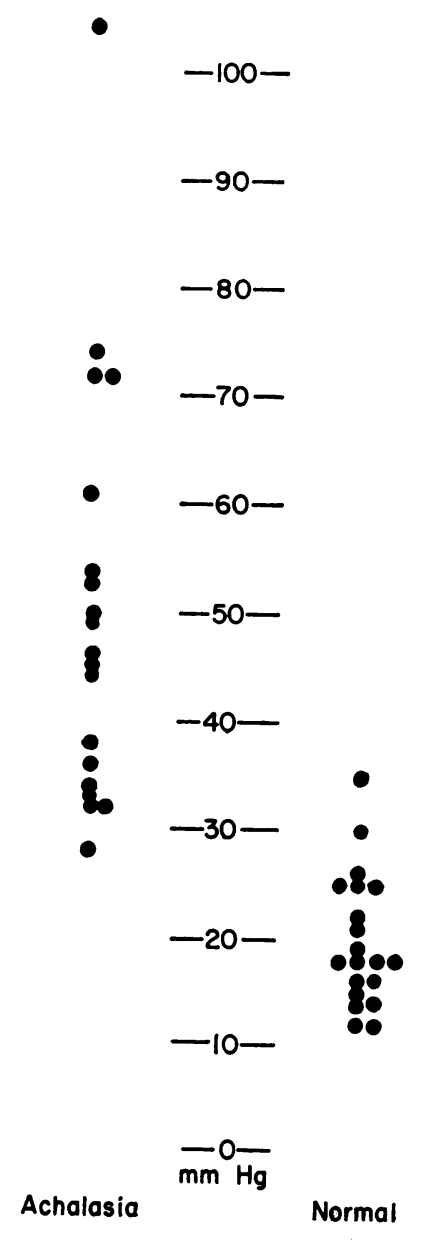

FigURE 1 LES pressures recorded in normals and patients with achalasia. Each point represents the mean of three measurements in an individual.

nostic evaluation, the recording assembly was positioned and anchored so that pressures were recorded simultaneously from the esophagus, LES, and stomach. The pressure recording tubes were infused with distilled water by a syringe pump at a constant rate of $1.2 \mathrm{ml} / \mathrm{min}$. LES pressures were recorded as millimeters of mercury with mean gastric fundic pressure used as the zero reference.

Hydrogen ion activity of the gastric contents was altered by instillation of acid ( $0.1 \mathrm{~N}$ hydrochloric acid), by constant infusion at a rate of $12 \mathrm{mEq} / 15 \mathrm{~min}$. This rate was adequate to keep an aspirated sample of gastric contents at $\mathrm{pH} 1.5$.

Gastrin $\mathrm{I}^{8}$ amino acid sequence 2-17 (Hexadecapeptide Amide), ${ }^{4}$ was given intravenously as a single $30 \mathrm{sec}$ injection through an indwelling anticubital catheter. Doseresponse curves were constructed from a single injection during a single study period. Multiple gastrin I injections were occasionally given on a single day, but successive injections were separated by a $60 \mathrm{~min}$ interval. The values

\footnotetext{
${ }^{8}$ FDA approval, IND 6363.

"Imperial Chemical Industries Ltd., Alderley Park, Cheshire, England.
}

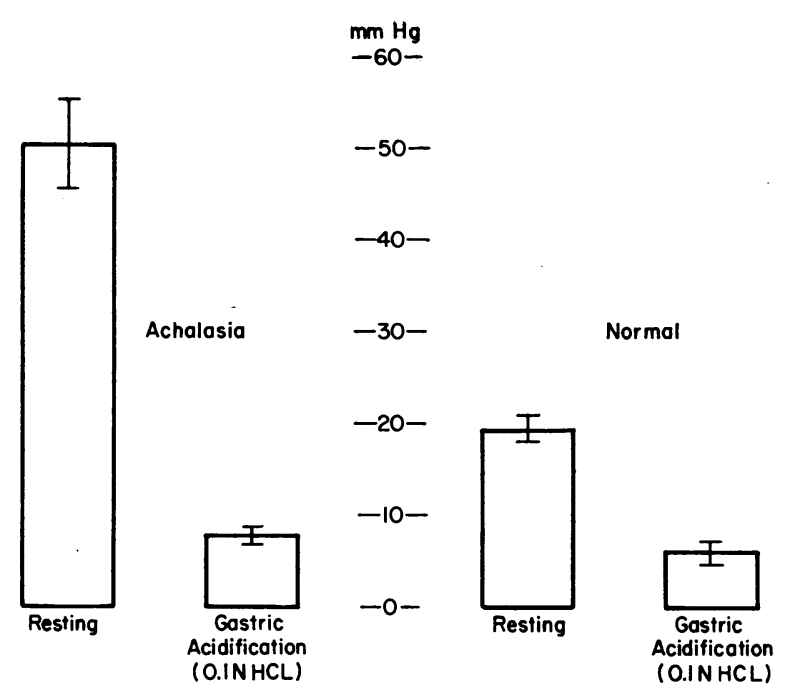

FIGURE 2 Comparison of the LES pressure recorded in all patients before and during gastric acidification with $0.1 \mathrm{~N}$ $\mathrm{HCl}$. The nadir of pressure attained during acidification is similar in both groups. The mean \pm 1 SEM is shown.

of pressure shown in the dose-response curves, are the mean values recorded over a 1 min interval.

Serum gastrin determinations were performed by the radioimmunoassay technique described by McGuigan (7-9). Blood was obtained at the time of manometric evaluation at least $20 \mathrm{~min}$ after passage of the recording assembly. Each gastrin determination was done in triplicate. The mean coefficient of variation of the triplicate values was $6.7 \%$.

\section{RESULTS}

In Fig. 1 are shown pressures recorded from 20 normal subjects and 19 untreated patients with achalasia. Each point represents the mean LES pressure obtained during the pull-through of the three recording orifices. Mean sphincter pressure in the normal group was $19.4 \pm 1.3$ $\mathrm{mm} \mathrm{Hg}$ (mean $\pm \mathrm{sE}$ ), as compared with $50.5 \pm 4.6 \mathrm{~mm}$ $\mathrm{Hg}$ in the patients with achalasia $(P<0.001)$. The range of pressures in untreated patients with achalasia was 28 to $108 \mathrm{~mm} \mathrm{Hg}$.

In Fig. 2 are shown LES pressures in normals and in patients with untreated achalasia recorded before and during gastric acidification with $0.1 \mathrm{~N} \mathrm{HCl}$ at $12 \mathrm{mEq} / 15$ min. Gastric acidification lowered LES pressure in normals from $19.4 \pm 1.3 \mathrm{~mm} \mathrm{Hg}$ to $6.1 \pm 1.2 \mathrm{~mm} \mathrm{Hg}$, a decrease of $68.4 \pm 3.1 \%$. In patients with achalasia, gastric acidification lowered pressure from $50.5 \pm 4.6 \mathrm{~mm} \mathrm{Hg}$ to $7.7 \pm 0.7 \mathrm{~mm} \mathrm{Hg}$, a decrease of $86.9 \pm 2.2 \%$. The nadir of pressure during gastric acidification was similar in normals and patients with achalasia $(P>0.05)$. The per cent reduction in pressure was significantly different $(P<0.001)$. 
To ascertain whether the high, acid-suppressible levels of LES pressure in patients with achalasia were due to increased levels of endogenous gastrin, serum gastrin levels were obtained on all patients in both groups. These results in Fig. 3 show no statistical difference between the two groups of patients $(P>0.05)$. In each group, normals and patients with achalasia, the LES pressure and serum gastrin level did not correlate $(P>$ 0.05).

Since LES hypertension in achalasia was not related to elevated endogenous levels of gastrin, the possibility of an increased sensitivity to gastrin was evaluated. In Fig. 4, dose-response curves of the LES to intravenous injections of synthetic gastrin I are shown. The peak LES response, averaged over a 1 min period, was expressed as a per cent of the initial base line LES pressure. Qualitative LES response was identical in both groups. The response began within $1 \mathrm{~min}$, peaked at 3 min, and dissipated to normal levels within $10 \mathrm{~min}$. Quantitatively, however, the response of the two groups was different. The dose-response curve in the patients with achalasia was shifted to the left, which indicates greater sensitivity to exogenous gastrin I. The threshold dose was lower in patients with achalasia. The steeply sloped linear portion of the dose-response curve, although shifted to the left, remained parallel to the comparable linear portion of the curve in the normal group. The peak response of the LES in patients with acha-

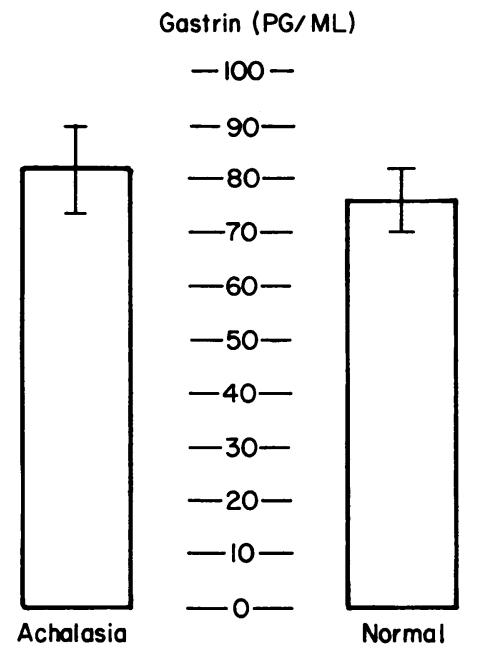

Figure 3 Comparison of serum gastrin levels in all patients at the time of recording LES pressure (Fig. 1). Mean and 1 SE are shown.

lasia was $433.4 \pm 14.7 \%$ of the preinjection level of LES pressure, and was attained at a dose of $0.25 \mu \mathrm{g} / \mathrm{kg}$ of gastrin I. The peak response of the LES in normals was $460.0 \pm 12.0 \%$ of the preinjection level of LES pressure, and was attained at $0.7 \mu \mathrm{g} / \mathrm{kg}$ of gastrin I. The magnitude of the peak response to gastrin I was similar in the two groups $(P>0.05)$, although the dose required to

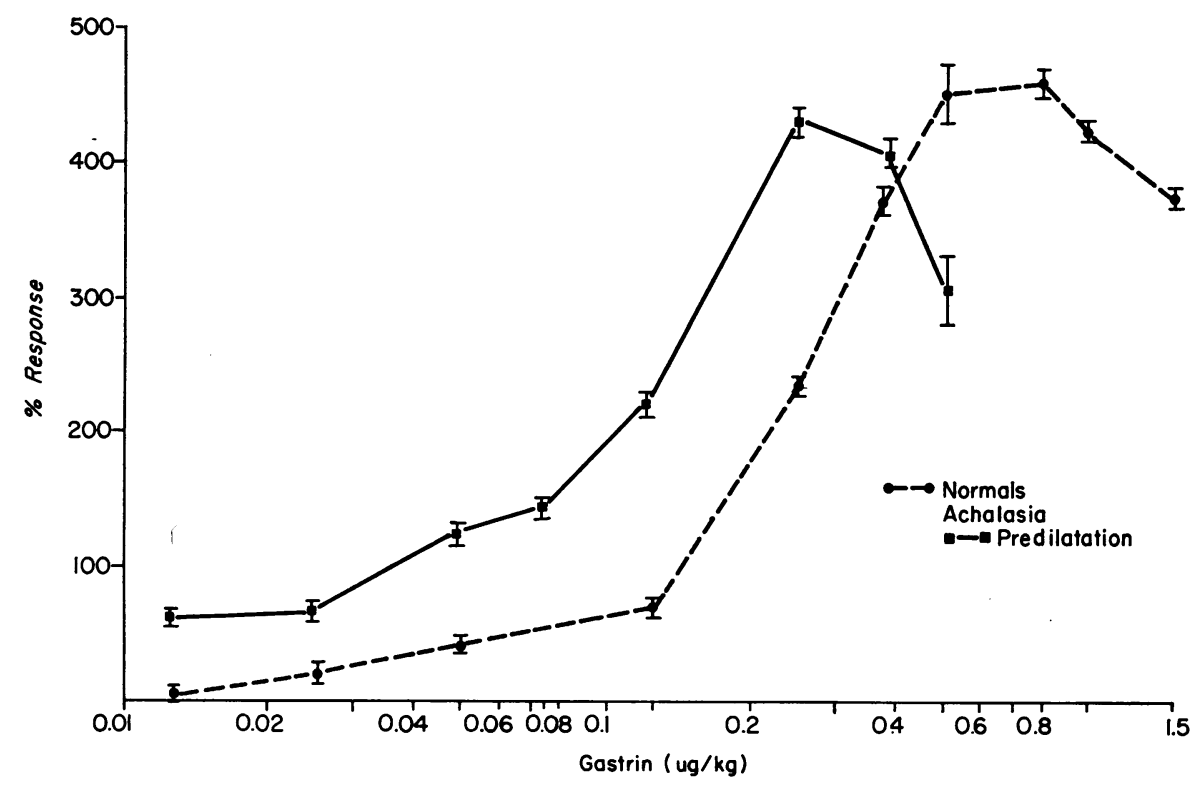

FIGURE 4 Dose-response curves of change in LES pressure, expressed as a per cent of initial resting sphincter pressure, against log dose of gastrin I in micrograms per kilogram. At each point is the mean \pm 1 SEM. Data are compiled from responses obtained in 13 normals and 11 patients with untreated achalasia. The LES in patients with achalasia is more sensitive to gastrin I, but not capable of a greater per cent response. 


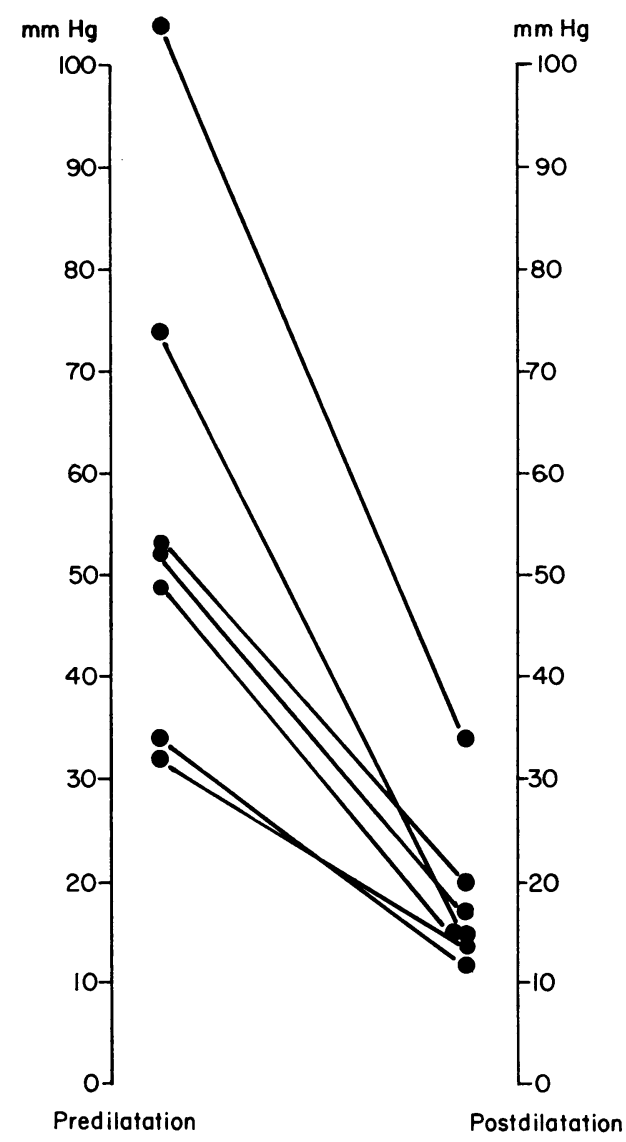

Figure 5 LES pressures recorded in seven patients with achalasia before and after a clinically successful pneumatic dilatation. LES pressure was significantly reduced in each patient after pneumatic dilatation.

attain this response was significantly different $(P<$ 0.001 ). It must be emphasized that although the peak per cent response is similar, the data is normalized for the preinjection level of LES pressure, as previously established (10). The absolute peak response in patients with achalasia is $183.6 \pm 26.9 \mathrm{~mm} \mathrm{Hg}$, as compared with $89.2 \pm 10.7 \mathrm{~mm} \mathrm{Hg}$ in the normal group. At doses of gastrin I beyond those which yielded peak responses the dose-response curves show a response significantly less than maximal. This phenomenon occurred at a lower dose, and to a more marked degree, in patients with achalasia than in the normal patients. At a dose of 0.5 $\mu \mathrm{g} / \mathrm{kg}$, patients with achalasia showed a response that was approximately $70 \%$ of the peak response at 0.25 $\mu \mathrm{g} / \mathrm{kg}$.

To ascertain whether the hypertensive initial LES pressure in patients with achalasia was responsible for the increased sensitivity to gastrin I, studies were repeated in patients with achalasia who had undergone a clinically and radiographically successful pneumatic dila- tation. ${ }^{5}$ In seven patients with achalasia, the mean LES graphic emptying of the esophagus.

pressure was lowered from $57.4 \pm 8.7 \mathrm{~mm} \mathrm{Hg}$ to 18.3 $\pm 3.0 \mathrm{~mm} \mathrm{Hg}$, after pneumatic dilatation $(P<0.001)$. The specific changes in resting LES pressure after pneumatic dilatation are shown in Fig. 5. All patients, except the one with an initial LES pressure of $108 \mathrm{~mm} \mathrm{Hg}$, were included in the dose-response curve shown in Fig. 4. Dose-response curves to gastrin I, shown in Fig. 6, are compared for the normals and for the patients with achalasia, pre-and postdilatation. The dose-response curve to gastrin $I$ in successfully treated patients with achalasia (i.e. normal sphincter pressure), still showed an increased sensitivity to exogenous gastrin I. Doseresponse curves to gastrin I were almost identical preand postdilatation.

\section{DISCUSSION}

These studies indicate that the LES in patients with achalasia, is (a) hypertensive, $(b)$ suppressed by gastric acidification, and (c) supersensitive to exogenous gastrin I. We propose that LES hypertension in patients with achalasia is due to supersensitivity to endogenous gastrin.

Cohen, using a constantly perfused recording system, has shown that LES pressure in patients with untreated achalasia is twice that of normal subjects $(3,11)$. His findings, confirmed in this study, differ from the normotensive levels of LES pressure previously reported (1, 2 ). This discrepancy was found to be the result of the methods employed in quantitating LES pressure. Prior studies were performed with uninfused open-tipped catheters, a method shown to be inadequate for recording the entire range of gastrointestinal sphincter pressure $(4,5)$. Harris and Pope have demonstrated that pressures recorded by this technique were influenced by mucosa sealing the recording orifice (12). This produced significant artifacts in interpreting LES pressure. The simple modification of measuring LES pressure with a constantly perfused recording system, has provided an accurate valid interpretation of the full range of LES strength (4-6).

Although the LES in patients with achalasia is hypertensive, gastric acidification consistently reduced the pressure as found in normals. This response of the LES was interpreted as a specific response to a reduction in endogenous gastrin released from the stomach. This interpretation was based on direct and indirect quantitation of the effect of gastric acidification on the release of gastrin in man (13) and animal models $(14,15)$. Comparison of the reduction in LES pressure to gastric acidification in both groups revealed a quantitative dif-

${ }^{5}$ (a) Symptomatic improvement in degree of dysphagia and nocturnal regurgitation, and $(b)$ improved cineradio- 


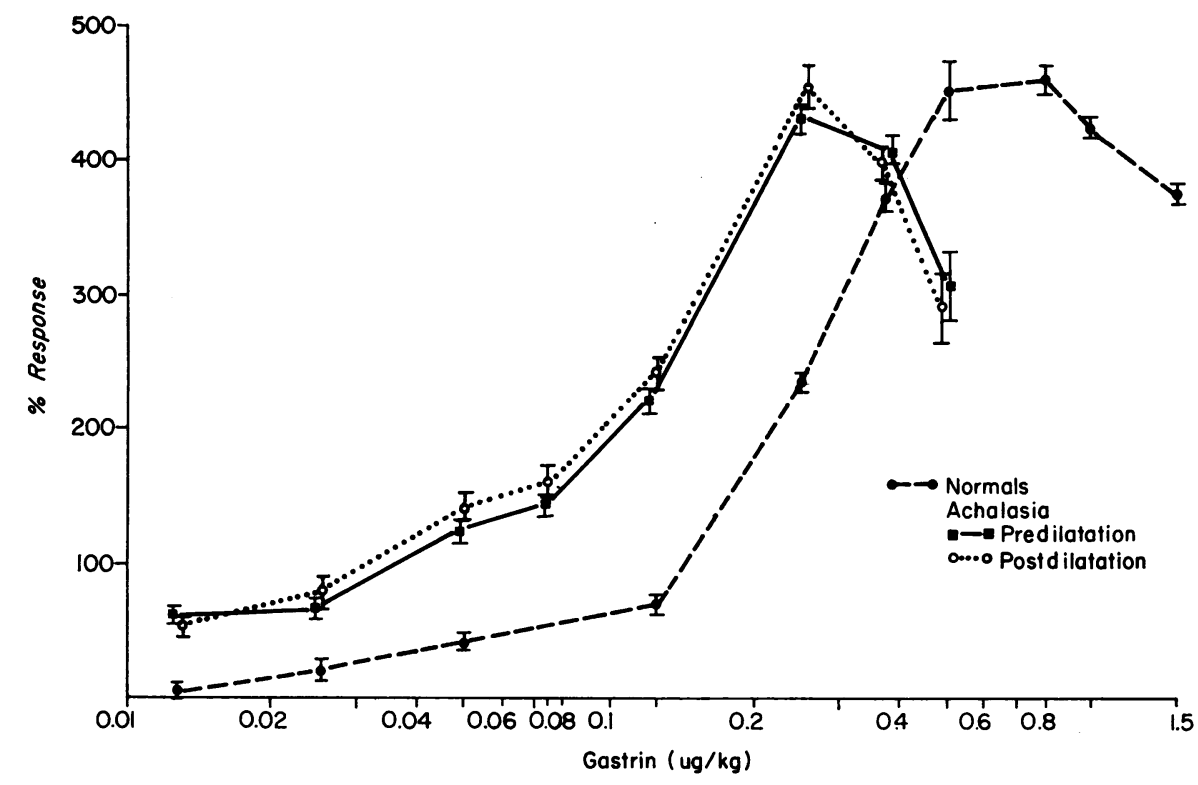

Figure 6 Dose-response curves of per cent change in LES pressure against log dose of gastrin $I$ in 13 normals, 11 patients with untreated achalasia, and 7 patients after pneumatic dilatation (Fig. 5). Patients with achalasia having a normal LES pressure after treatment, still show supersensitivity to gastrin I.

ference in response. A greater absolute and a greater per cent reduction in pressure was found in the achalasic group. The nadir of pressure attained during inhibition was the same in the two groups. This finding suggested that a greater portion of the LES pressure in patients with achalasia was determined by endogenous gastrin. The portion of LES pressure that was not acid suppressible and, therefore, not gastrin dependent, did not differ in the two groups. This large, gastrin-related portion of LES pressure in patients with achalasia suggested two possibilities: (a) either endogenous levels of gastrin were increased, or $(b)$ the LES was supersensitive to endogenous gastrin. The first possibility was proven incorrect by demonstrating that patients with achalasia had normal levels of serum gastrin. This possibility initially seemed unlikely since patients with elevated levels of gastrin, as in pernicious anemia (16) and Zollinger-Ellison syndrome, ${ }^{8}$ have normal or diminished resting levels of LES pressure.

To evaluate the second possibility, dose-response curves of LES pressure to exogenous gastrin I were compared in normals and in patients with untreated achalasia. Gastrin supersensitivity was found in the latter group. This finding was established by showing a lower threshold dose, a leftward parallel shift in the linear portion of the response, and the absence of change in

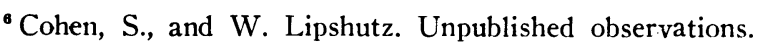

magnitude of the maximum per cent response. The achalasic sphincter, although more sensitive to gastrin, did not show a greater per cent increase in pressure than the normal sphincter. These characteristics of supersensitivity are identical with those of Cannon $(17,18)$ and Trendelenburg (19), who demonstrated these findings with experimentally denervated animal models.

Although the maximum per cent increase in LES pressure was similar in both groups, patients with achalasia had a greater absolute increase in LES pressure. This finding was related to the greater initial LES pressure in achalasia. Expression of data as a per cent of initial pressure normalizes for baseline values and allows for quantitation of dose-response relationships. When the LES pressure of a normal was elevated to the achalasic range, the maximum absolute change in pressure was similar to that of the patient with achalasia. Similarly, when the LES pressure in patients with achalasia was reduced to a normal level by pneumatic dilatation, the dose-response curve was still shifted to the left, but now the absolute LES response was similar to that obtained in normals.

Further examination of the dose-response curves revealed another possible manifestation of supersensitivity. At points beyond the maximal response, autoinhibition or the diminished response of a system to doses beyond that which yielded a maximum response, was observed. 
It is due to saturation of all high affinity excitatory receptors with additional molecules combining with a low affinity inhibitory receptor (20). Whereas this phenomenon, was observed in both groups, it occurred at lower doses and to a more marked degree in patients with achalasia. This may result from supersensitivity of inhitory as well as excitatory receptors.

Denervation supersensitivity of the body of the esophagus has been generally accepted as a clinical criteria for the diagnosis of achalasia (21). However, the LES has not been studied for this property. Anatomic evidence of denervation has been shown in the sphincter zone (22), but this loss of myenteric ganglia has not been consistently observed $(23,24)$. Although conclusive evidence demonstrating denervation of the achalasic LES is not available, this study does establish supersensitivity to gastrin I. We propose that this supersensitivity is due to denervation. There are several mechanisms by which the achalasic LES could become supersensitized to gastrin. Cannon has shown that denervation leads to supersensitivity not only to natural neural transmitters, but also, nonspecifically, to other chemical stimulants (18). Gastrin supersensitivity may, therefore, be a nonspecific reaction of a denervated structure. A second possible mechanism of gastrin supersensitivity involves an intermediate site of action of gastrin in the control of LES strength. It has been shown that gastrin effect on smooth muscle is through postganglionic release of acetylcholine (25). Studies on the circular smooth muscle of the opossum LES in vitro confirm this mechanism of action. ${ }^{7}$ Therefore, supersensitivity may be due to the neural transmitter, acetylcholine, not directly to the peptide hormone. Regardless of the specific mechanism of gastrin supersensitivity, the significance of this finding is not altered. In achalasia, the marked acid suppression of the elevated LES pressure is due to inhibition of endogenous gastrin release. Therefore, it is suggested that the elevated LES pressure is due to supersensitivity to endogenous gastrin.

The demonstration of lower esophageal sphincter hypertension and an elucidation of its pathophysiology are initial steps in searching for a medical program for treating achalasia. If the sphincter can partially relax, as shown by Cohen (3) and confirmed by us, then the sphincter-to-stomach gradient is dependent upon both the level of sphincter pressure from which the relaxation occurs and the degree of sphincter relaxation. The correlation between clinical improvement and reduction in sphincter pressure indicates that if LES pressure were lowered through inhibition of gastrin effect, then achalasia might be treated medically.

${ }^{7}$ Lipshutz, W., and S. Cohen. Unpublished observations.

\section{ACKNOWLEDGMENTS}

The authors acknowledge the technical assistance of Mrs. Anne Cohen.

This research was supported by Research Associate and Training Grant (TR-15A) funds through the Veterans Administration, Clinical Research Center Grant 5-M01RR40-09, Division of Research Facilities and Resources, National Institutes of Health and Training Grant T01AM5462-06, U. S. Public Health Service.

\section{REFERENCES}

1. Vantrappen, G., G. E. Van Goidsenhoven, S. Verbeke, G. Van Den Berghe, and J. Vandenbroucke. 1963. Manometric studies in achalasia of the cardia, before and after pneumatic dilations. Gastroenterology. 45: 317.

2. Creamer, B., A. M. Olsen, and C. F. Code. 1957. The esophageal sphincters in achalasia of the cardia (cardiospasm). Gastroenterology. 33: 293.

3. Cohen, B. R. 1965. "Cardiospasm" in achalasia : demonstration of an abnormally elevated esophagogastric sphincter pressure with partial relaxation on swallowing. Gastroenterology. 48: 464. (Abstr.)

4. Winans, C. S., and L. D. Harris. 1967. Quantitation of lower esophageal sphincter competence. Gastroenterology. $52: 773$.

5. Pope, C. E., II. 1967. A dynamic test of sphincter strength: it's application to the lower esophageal sphincter. Gastroenterology. 52: 779.

6. Cohen, S., and L. D. Harris. 1970. Lower esophageal sphincter pressure as an index of lower esophageal sphincter strength. Gastroenterology. 58: 157.

7. McGuigan, J. E. 1967. Antibodies to the carboxy-terminal tetrapeptide of gastrin. Gastroenterology. 53: 697 .

8. McGuigan, J. E. 1968. Immunochemical studies with synthetic human gastrin. Gastroenterology. 54: 1005.

9. McGuigan, J. E., and W. L. Trudeau. 1970. Studies with antibodies to gastrin. Radioimmunoassay in human serum and physiological studies. Gastroenterology. 58: 139.

10. Cohen, S., and W. Lipshutz. 1971. Hormonal regulation of human lower esophageal sphincter competence: interaction of gastrin and secretin. J. Clin. Invest. 50: 449.

11. Pope, C. E., II. 1970. The esophagus: 1967-1969. Gastroenterology. 59: 460.

12. Harris, L. D., and C. E. Pope II. 1964. "Squeeze" vs, resistance: an evaluation of the mechanism of sphincter competence. J. Clin. Invest. 43: 2272.

13. Yalow, R. S., and S. A. Berson. 1970. Radioimmunoassay of gastrin. Gastroenterology. 58: 1 .

14. Grossman, M. I., editor. 1966. Gastrin. University of California Press, Berkeley.

15. Andersson, S., and L. Olbe. 1964. Inhibition of gastric acid response to sham feeding in Pavlov pouch dogs by acidification of antrum. Acta Physiol. Scand. 61: 55.

16. Castell, D. O., and L. D. Harris. 1970. Hormonal control of gastroesophageal-sphincter strength. N. Engl. J. Med. 282: 886.

17. Cannon, W. B., and A. Rosenblueth. 1949. The Supersensitivity of Denervated Structures. The Macmillan Company, New York.

18. Cannon, W. B. 1939. A law of denervation. Amer. J. Med.Sci. 198: 737. 
19. Trendelenburg, U. 1966. I. Mechanisms of supersensitivity and subsensitivity to sympathomimetic amines. Pharmacol. Rev. 18: 629.

20. Ariëns, E. J., editor. 1964. Molecular Pharmacology. Vol. I. Academic Press Inc., New York.

21. Kramer, P., and F. I Ingelfinger. 1951. Esophageal sensitivity to mecholyl in cardiospasm. Gastroenterology. 19: 242.

22. Misiewicz, J. J., S. L. Waller, P. P. Anthony, and J. W. Gummer. 1969. Achalasia of the cardia: pharmacology and histopathology of isolated cardiac sphincteric muscle from patients with and without achalasia. Quart. J. Med. 38: 17 .

23. Cassella, R. R., A. L. Brown, Jr., G. P. Sayre, and F. H. Ellis, Jr. 1964. Achalasia of the esophagus: pathologic and etiologic considerations. Ann. Surg. 160: 474.

24. Trounce, J. R., D. C. Deuchar, R. Kauntze, and G. A. Thomas. 1957. Studies in achalasia of the cardia. Quart. J. Med. 26: 433.

25. Bennett, A. 1965. Effect of gastrin on isolated smooth muscle preparations. Nature (London). 208: 170. 